RAEIC, Revista de la Asociación Española de Investigación de la Comunicación

vol. 7 , núm. 13 (2020), 290-294

ISSN 2341-2690

DOI: https://doi.org/10.24137/raeic.7.13.14

\title{
Reseñas 1
}

\section{Book Reviews 1}

Legerén-Lago, Beatriz

Universidade de Vigo (UVIGO)

blegeren@uvigo.es

\section{Forma de citar este artículo:}

Legerén-Lago, B. (2020). "Reseñas 1", RAEIC, Revista de la Asociación Española de Investigación de la Comunicación, vol. 7, núm. 13, 290-294.

https://doi.org/10.24137/raeic.7.13.14 


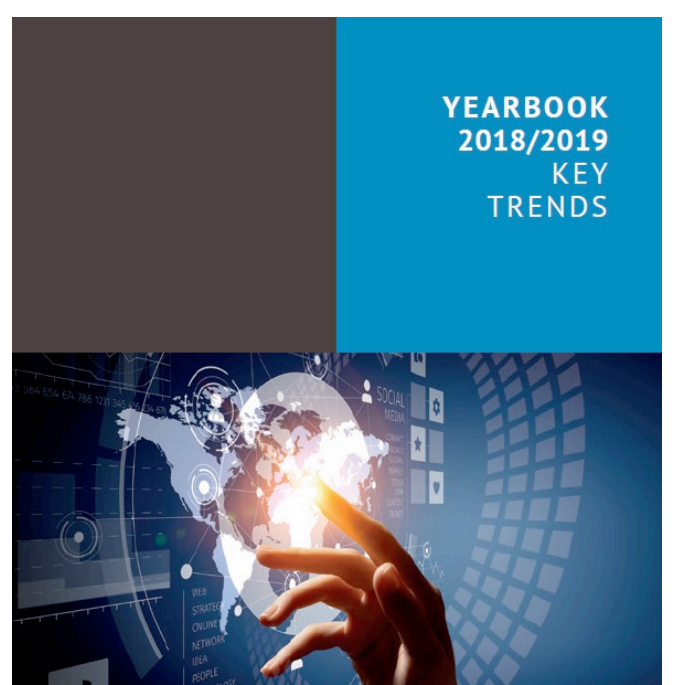

Título: Yearbook 2018/2019 Key Trends. Television, cinema, Video and on-demand audiovisual services. The pan European picture.

Autores: Francisco Javier Cabrera Blázquez, Maja Cappello, Léa Chochon, Laura Ene, Gilles Fontaine, Christian Grece, Marta Jiménez Pumares, Martin Kanzler, Ismail Rabie, Agnes Schneeberger, Patrizia Simone, Julio Talavera, Sophie Valais.

Editorial: European Audiovisual Observatory

Páginas: 72

URL: https://rm.coe.int/yearbook-keytrends-2018-2019-en/1680938f8e

El Observatorio Audiovisual Europeo, es una institución creada en el año 1992 al objeto de responder a una falta de información y transparencia sobre esta industria. Su principal objetivo es proporcionar una visión europea comparativa de la industria audiovisual en 41 países diferentes, así como un análisis detallado de las industrias nacionales e incluso regionales, y es el responsable del Anuario de tendencias del audiovisual.

Esta cuarta edición del Anuario de tendencias nos muestra una gama de ideas y datos comparando el mercado audiovisual europeo, formado por un mosaico de realidades nacionales, versus el mercado norteamericano construido "de abajo hacia arriba", desde estaciones locales de televisión hasta servicios audiovisuales nacionales.

El trabajo realizado por el equipo de analistas y compilado este anuario pone de relieve, interesantes diferencias y similitudes resultado de la comparación de las cifras clave de las dos áreas analizadas. 
Los contenidos de este informe abarcan todo el modelo del negocio de la industria audiovisual, tanto las áreas producción, distribución y servicios audiovisuales como mercados y participantes.

Comienza trabajando sobre producción tanto de cine como de televisión. Mientras los datos de la industria cinematográfica confirman que se está produciendo un estancamiento debido al descenso en las inversiones, ya que los principales soportes siguen siendo la financiación pública y los emisores, en Rusia se está produciendo un alza en la misma. Los de la televisión muestran

Otro de los temas tratados en esta sección es la necesidad de modernizar las leyes de protección de derechos, y también muestra los datos sobre la situación de género en la industria audiovisual.

En las secciones de distribución y servicios audiovisuales destacan la penetración de las plataformas digitales al tiempo que resalta que un $21 \%$ de las películas de SVOD son europeas. Paradójicamente, al tiempo que la televisión lineal decrece, aumentan los canales de noticias en Europa siendo estos de varios propietarios y presentan ofertas variadas.

En el apartado de mercados, los datos muestran que la publicidad se encuentra frente al reto que presentan los anuncios on line, al mismo tiempo que la televisión lineal debe pelear con el incremento de la IPTV y el explosivo crecimiento de los servicios de suscripción de video bajo demanda.

Pero, es la sección de participantes la que presenta más relevancia de cara a la investigación en la estamos trabajando en la actualidad. Ya que señala que las emisoras de servicio público (PSB) están viendo disminuir los recursos financieros debido a los multimillonarios presupuestos de empresas como Amazon y Netflix que están invirtiendo en contenido local, y está competencia está impulsando a las redes locales a aumentar la producción de contenido original. Pero no sólo eso, sino que además obliga a las PSBs europeas a colaborar no sólo en términos de contenido, sino también en convergencia de plataformas. Estimulando las alianzas entre emisoras públicas y privadas en un intento por mantenerse y competir los servicios de video on-demand. 
Por otra parte, los datos de este estudio confirman el cambio en el consumo de video por parte de los adolescentes, púes lo hacen más a través de internet, bien a través de plataformas creadas para compartir videos (Youtube, Vimeo...), como de redes sociales. En este último caso distinguen dos categorías, en primer lugar, las que han incorporado la posibilidad de compartir videos (Facebook, Instagram...) y en segundo lugar las creadas a tal objeto Twitch, Persicope, live.me... Y aunque ambos modelos continúan, los operadores no han trabajado con ellos. Puesto que el intercambio de videos, sólo se utiliza como apoyo a la actividad principal de estos.

Este informe nos facilita una radiografía de la situación del panorama audiovisual paneuropeo en la actualidad.

Su lectura y análisis nos ayuda a entender la situación en la que se encuentra en la actualidad y puede dar pautas sobre cómo trabajar y enfocarnos hacia el futuro en el sector audiovisual europeo.

Como se puede ver según los datos mostrados, la globalización está afectando a los contenidos. La audiencia tiene capacidad para escoger qué ver, cuándo y cómo. Y esta multiplicación de opciones no ha hecho más que incrementar la competencia de los operadores para atraer a unas audiencias cada vez más desligadas de la linealidad televisiva. (Crespo-Pereira, Verónica; Legeren-Lago, 2019)

Por otra parte, la digitalización al igual que democratiza los medios de producción y distribución, obliga a trabajar a las empresas de contenidos con empresas de tecnología, pues todo está convergiendo y las PSM deben hacerlo en igual medida. (Legeren Lago, 2019).

\section{REFERENCIAS}

Crespo-Pereira, V., y Legeren-Lago, B. (2019). Estrategias de la televisión pública europea ante la oferta informativa en la era digital. El caso de la televisión pública británica. Revista Ibérica de Sistemas e Tecnologias de Informação Iberian Journal of 
Information Systems and Technologies, E20, 1-739. Recuperado de http://www.risti.xyz/issues/ristie20.pdf

European Commission. (2018). Audiovisual Media Services Directive (AVMSD) | Digital Single Market. Recuperado de https://ec.europa.eu/digital-single-

market/en/audiovisual-media-services-di

Legeren Lago, B., y Zagalo, N. (2019). Diseño de Mundos-Historia. Revista ICONO14

Revista Científica De Comunicación Y Tecnologías Emergentes, 17(1), 1-4.

https://doi.org/10.7195/ri14.v17i1.1306

Observatory, E. A. (n.d.). Iris newsletter. Recuperado de https://merlin.obs.coe.int/newsletter/index

Observatory, E. A. (n.d.). LUMIERE: Search. Recuperado de http://lumiere.obs.coe.int/web/search/

Observatory, E. A. (n.d.). Mavise - Home. Recuperado de http://mavise.obs.coe.int/

\section{AGRADECIMIENTOS}

Este artículo forma parte de las actividades del proyecto de investigación (RTI2018096065-B-100) del Programa Estatal de I+D+l orientado a los Retos de la Sociedad del Ministerio de Ciencia, Innovación y Universidades (MCIU), Agencia Estatal de Investigación (AEI) y del Fondo Europeo de Desarrollo Regional (FEDER) sobre "Nuevos valores, gobernanza, financiación y servicios audiovisuales públicos para la sociedad de Internet: contrastes europeos y españoles". 